\title{
Energy Efficient Control Strategies in Heterogeneous Wireless Sensor Networks: A Survey
}

\author{
Shilpa Mahajan \\ Computer Science Department, ITMU \\ Sec-23 A, Palam Vihar, Gurgaon (India)
}

\author{
Dr. Jyoteesh Malhotra \\ Electronics and Communication Department, \\ GNDU Regional campus, Jalandhar (India)
}

\begin{abstract}
Wireless sensor networks (WSNs) have received tremendous attention in recent years because of the development of sensor devices, as well as wireless communication technologies. It is usually randomly deployed in inaccessible terrains, disaster areas, or polluted environments, where battery replacement or recharge is difficult or even impossible to be performed. For this reason, network lifetime is of crucial importance to a WSN. In this article we present a survey of various energy efficient techniques in a heterogeneous wireless sensor network .We first outline the basic network radio model and how this model can be used to study various trades off between network deployment costs, clustering approach in terms of energy efficiency. We also high lights some energy efficient protocols that can be used in heterogeneous networks.
\end{abstract}

\section{Keywords:}

Heterogeneous protocols, Network life time, Cost, Energy conservation

\section{INTRODUCTION}

In a WSN, sensor nodes are typically operated by batteries, which are limited in energy capacity, and difficult or even impossible to be replaced or recharged. For this reason, power control is needed to efficiently make use of the limited energy resources in order to minimize the energy consumed by the sensor nodes and thus prolong network lifetime. For this purpose, energy efficiency must be considered in every aspect of network design and operation, not only for individual sensor nodes, but also for the communication of the entire network. Energy efficiency and power control are the basic guarantee of the network performance, for example, throughput and delay.

In this article we present a survey of protocols and scheme used in heterogeneous networks .Our aim is to provide a better understanding of the current issues in this emerging field for energy conservation.

\subsection{Power Consumption}

The wireless sensor network node being electronic device can only be equipped with limited power source. A node structure as shown in figure 1 typically consist of four basic components: a sensing unit, a processing unit, a communication unit, and a power unit [1].

Sensor node lifetime therefore shows a strong dependency on battery malfunctioning of a few nodes and can cause significant topological change and might require re-routing of packets and reorganisation of the network. Hence, power conservation and power management takes an additional importance. It is for these reasons that researchers are currently focussing on the design of power aware protocols and algorithms for sensor network.

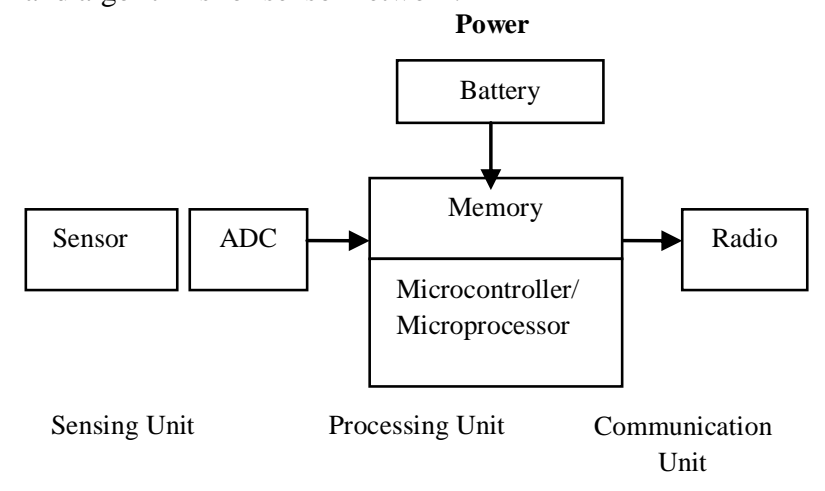

Fig 1: Sensor node structure

\section{HETEROGENEOUS MODEL FOR WSN}

Most of the protocols designed for WSNs assume that the sensors have the same capabilities in terms of storage, processing, sensing, and communication. The resulting network is said to be homogeneous. In these types of networks, a pair of sensors would have the same lifetime if they have the same energy consumption rate. Some sensing applications, however, use sensors with different capabilities and accordingly the resulting network is said to be heterogeneous. In the real world, the assumption of homogeneous sensors may not be practical because sensing applications may require heterogeneous sensors in terms of their sensing and communication capabilities in order to enhance network reliability and extend network lifetime [2] Also, even if the sensors are equipped with identical hardware, they may not always have the same communication and sensing models. In fact, at the manufacturing stage, there is no guarantee that two sensors using the same platform have exactly the same physical properties. This taxonomy focuses on heterogeneity at the designing stage, when sensors are designed to have non identical capabilities to meet the specific needs of sensing applications.

In this section, we will present a paradigm of heterogeneous wireless sensor network and discuss the impact of heterogeneous resources.

There are three common types of resource heterogeneity in sensor node computation -al heterogeneity, link heterogeneity, and Energy Heterogeneity [3]. 
Computational heterogeneity means that the heterogeneous node has a more powerful microprocessor and more memory than the normal node.

Link heterogeneity means that the heterogeneous node has high-bandwidth and long-distance network transceiver than the normal node. Link heterogeneity can provide more reliable data transmission.

Energy heterogeneity means that the heterogeneous node is line powered, or its battery is replaceable. Among above three types of resource heterogeneity, the most important heterogeneity is the energy heterogeneity because both computational heterogeneity and link heterogeneity will consume more energy resource. If there is no energy heterogeneity, computational heterogeneity and link heterogeneity will bring negative impact to the whole sensor network, i.e., decreasing the network lifetime.

\subsection{Heterogeneous impact on the wireless sensor networks}

Placing few heterogeneous nodes in the sensor network can bring following three main benefits:

1. Prolonging network lifetime. In the heterogeneous wireless sensor network, the average energy consumption for forwarding a packet from the normal nodes to the sink in heterogeneous sensor networks will be much less than the energy consumed in homogeneous sensor networks.

\subsection{Performance measures}

We define here the measures that can be used to evaluate the performance of heterogeneous.

Network lifetime: This is the time interval from the start of operation (of the sensor network) until the death of the first alive node.

Number of cluster heads per round: This instantaneous measure reflects the number of nodes which would send directly to the sink information aggregated from their cluster members.

Number of alive (total, super, advanced and normal) nodes per round: This instantaneous measure reflects the total number of nodes and that of each type that has not yet expended all of their energy.

Throughput: We measure the total rate of data sent over the network, the rate of data sent from cluster heads to the sink as well as the rate of data sent from the nodes to their cluster heads.

\section{ENERGY AWARE CONTROL STRATEGIES IN HETEROGENEOUS NETWORK}

A sensor network is composed of a large number of sensor nodes and a sink. The base station typically serves as a gateway to some other networks. It provides powerful data processing, storage centre, and an access point to the sensor nodes in its network.

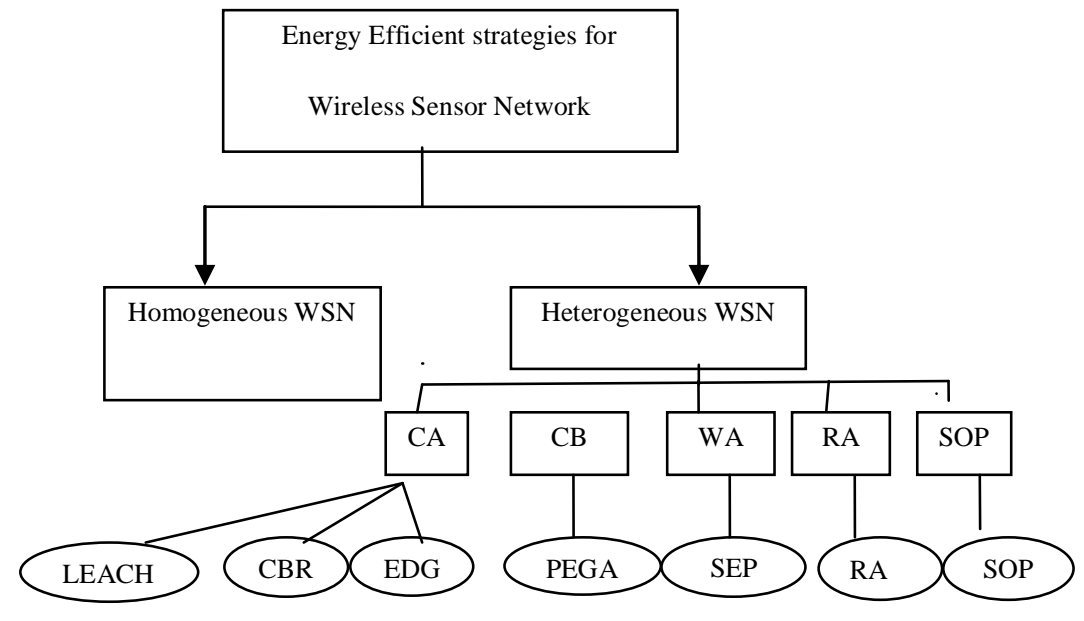

\author{
$\mathrm{CB}=$ Chain Based \\ $\mathrm{CA}=$ Clustering Approach \\ WA=Weight Assignment \\ $\mathrm{RA}=$ Randomized Approach
}

Fig 2: Taxonomy of Energy Efficient Strategies of heterogeneous WSN

2. Improving reliability of data transmission. It is well known that sensor network links tend to have low reliability. And each hop significantly lowers the end-to-end delivery rate. With heterogeneous nodes; there will be fewer hops between normal sensor nodes and the sink. So the heterogeneous sensor network can get much higher end-to-end delivery rate than the homogeneous sensor network.

3. Decreasing latency of data transportation. Computational hetero-geneity can decrease the processing latency in immediate nodes. And link heterogeneity can decrease the waiting time in the transmitting queue. Fewer hops between sensor nodes and sink node also mean fewer forwarding latency.
Sensor nodes sense their environment, collect sensed data and transmit it to the BS. However, they are limited in power, computational capacity and memory. Placing few heterogeneous nodes in wireless sensor network is an effective way to increase network lifetime and reliability. Various energy efficient heterogeneous schemes have been discussed in figure 2 .

\subsection{Cluster based approach}

In a hierarchical network, sensor nodes are organized into clusters, where the cluster members send their data to the cluster heads while the cluster heads serve as relays for transmitting the data to the sink. A node with lower energy can be used to perform the sensing task and send the sensed data to its cluster head at short distance, while a node with 
higher energy can be selected as a cluster head to process the data from its cluster members and transmit the processed data to the sink. This process can not only reduce the energy consumption for communication, but also balance traffic load and improve scalability when the network size grows. The major problem with clustering is how to select the cluster heads and how to organize the clusters [4]. In this context, there are many clustering strategies.

There are certain the set of attributes that can be use to categorizes and differentiate clustering algorithms of WSN [20]. The following attributes of the $\mathrm{CH}$ node are differentiating factors among clustering schemes:

- Mobility: When a $\mathrm{CH}$ is mobile, sensor's membership dynamically changes and the clusters would need to be continuously maintained. On the other hand, stationary $\mathrm{CH}$ tends to yield stable clusters and facilitate intra- and intercluster network management

- Node types: In some setups a subset of the deployed sensors are designated as $\mathrm{CHs}$ while in others $\mathrm{CHs}$ are equipped with significantly more computation and communication resources. - Role: A CH can simply act as a relay for the traffic generated by the sensors in its cluster or perform aggregation/ fusion of collected sensors' data. Sometime, a CH acts as a sink or a base-station that takes actions based on the detected phenomena or targets.

Depending on the objective and the methodology, numerous clustering algorithms have been proposed. The complexity and convergence rate of these algorithms can be constant or dependent on the number of $\mathrm{CHs}$ and/or sensors.

Low-energy adaptive clustering hierarchy (LEACH) [5] is one of the most popular distributed cluster-based routing protocols in wireless sensor networks.

LEACH randomly selects a few nodes as cluster heads and rotates this role to balance the energy dissipation of the sensor nodes in the networks. The cluster head nodes fuse and aggregate data arriving from nodes that belong to the respective cluster. And cluster heads send an aggregated data to the sink in order to reduce the amount of data and transmission of the duplicated data. Data collection is centralized to sink and performed periodically. The operation of LEACH is generally separated into two phases, the set-up phase and the steady-state phase. In the set-up phase, cluster heads are selected and clusters are organized. In the steadystate phase, the actual data transmissions to the sink take place. After the steady-state phase, the next round begins.

$$
\text { pnrm }=\frac{\text { popt }}{1+\alpha . \mathrm{m}} \mathrm{T}(\mathrm{s})=\left\{\begin{array}{l}
\frac{\mathrm{popt}}{1-\text { popt } *\left(\mathrm{r} * \bmod \frac{1}{\text { popt }}\right)} \\
0 \quad \text { otherwise }
\end{array}\right\}
$$

During the set-up phase, when clusters are being created, each node decides whether or not to become a cluster head for the current round. This decision is based on a predetermined fraction of nodes and the threshold $\mathrm{T}(\mathrm{s})$. The threshold is given by Equation (1) [4] where $p_{\text {opt }}$ is the predetermined percentage of cluster heads (e.g., popt $=0.05$ ), $r$ is the current round, and $G$ is the set of nodes that have not been cluster heads in the last 1/popt rounds. Using this threshold, each node will be a cluster head at some round within 1/popt rounds. After $1 / \mathrm{p}_{\mathrm{opt}}$ rounds, all nodes are once again eligible to become cluster heads. In LEACH, the optimal number of cluster heads is estimated to be about $5 \%$ of the total number of nodes. Each node that has elected itself cluster head for the current round broadcasts an advertisement message to the rest of the nodes in the network.

All the non-cluster head nodes, after receiving this advertisement message, decide on the cluster to which they will belong for this round. This decision is based on the received signal strength of the advertisement messages. After cluster head receives all the messages from the nodes that would like to be included in the cluster and based on the number of nodes in the cluster, the cluster head creates a TDMA schedule and assigns each node a time slot when it can transmit.

During the steady-state phase, the sensor nodes can begin sensing and transmitting data to cluster heads. The radio of each non cluster head node can be turned off until the node's allocated transmission time. The cluster heads, after receiving all the data, aggregate it before sending it to the sink. Each cluster head communicates using different CDMA codes in order to reduce interference from nodes belonging to other clusters.

\subsubsection{Measures suggested as improvement in cluster based heterogeneous network}

A considerable amount of research have been done in this area and simulation results shows that by applying various energy control strategies, considering different parameters , an effective results can be obtained.

A self organizing clustering algorithm CODA i.e. Clusterbased self-Organizing Data Aggregation method based on the distance from the sink and an aggregating data using competitive machine learning [6]. CODA divides the whole network into a small number of groups based on the distance from the base station and the strategy of routing and each group has its own number of cluster members and member nodes. The farther the distance from the base station, the more clusters are formed in case of single hop as shown in figure 4. with clustering. It shows better performance than applying the same probability to the whole network in terms of the network lifetime and the dissipated energy.

In [7], the authors discuss a clustering algorithm which periodically selects cluster head based on the node residual energy and node degree and a secondary parameter, such as node proximity to its neighbours or node degree. The clustering process terminates in $O(1)$ iterations and it also achieves fairly uniform cluster head distribution across the network and selection of the secondary clustering parameter can balance load among cluster heads.

In [8] the authors introduce a cluster head election method using fuzz logic to overcome the defects of LEACH. They inquired that the network lifetime can be prolonged by using fuzz variables in homogeneous network system, which is different from the heterogeneous energy consideration. The two parameters considered are location and energy of each sensor node.

In [9] the authors propose an EDGA algorithm to achieve good performance in terms of lifetime by minimizing energy consumption for in -network communications and balancing the energy load. It is based on weighted election probabilities of each node to become a cluster head, which can better handle the heterogeneous energy capacities and adopt a 
simple but efficient method to solve the area coverage problem in a cluster range.

Recently, in [10,11], authors suggested the impact of heterogeneity of nodes in terms of their energy that are hierarchically clustered in WSNs and initiate an energy efficient heterogeneous clustered method for WSNs based on weighted election probabilities of each node to become a cluster head according to the residual energy in each node. For this they suppose a percentage of the population of sensor nodes is equipped with the additional energy resources.

In CBRP(Clustered based routing hierarchal routing protocol, a new concept called headset, consist of one active cluster head and some other associate cluster heads with in the cluster [12]. The head set members are responsible for control and management of the network the head set is responsible to send message to the base station. results shows that this protocol performance better as compare to LEACH in context to energy consumption, frame transmission, and lifetime of the network.

RCFT(Re-clustering formation technique) suggested is to disperse and re organise cluster heads considering number of hops between clusters organised randomly and the belonging nodes for the sake of the efficient division of clusters. This technique aims to elect cluster head efficiently which has a direct impact on energy consumption [13].

The goal of SEP ( Stable Election Protocol) Protocol is to increase the stable region and as a result decrease the unstable region and improve the quality of the feedback of wireless clustered sensor networks, in the presence of heterogeneous nodes .In order to prolong the stable region, SEP attempts to maintain the constraint of well balanced energy consumption [15]. Two types of nodes are considered, a fraction of advanced nodes $(\mathrm{m})$ and the additional energy factor between advanced and normal nodes

A HWSNs (Heterogeneous wireless sensor network model) based on energy and computational heterogeneity [19].EDFM is a self-adaptive clustering routing protocol similar with LEACH. Different from other energy efficient protocols which consider the residual energy and energy consumption rate of nodes, cluster heads election in EDFM is based on one step energy consumption forecast method [20]. The algorithm tries to balance energy consumption round by round, which will provide the longest stable period for the networks.

Random competition based clustering (RCC): Although RCC [21] is designed for mobile ad hoc networks, it is also applicable to WSNs. RCC mainly focuses at cluster stability in order to support mobile nodes. The RCC algorithm applies the First Declaration Wins rule, in which any node can "govern'" the rest of the nodes in its radio coverage if it is the first to claim being a $\mathrm{CH}$.

\subsection{Chain based approach}

The main idea in PEGASIS [14] is for each node to receive from and transmit to close neighbours and take turns being the leader for transmission to the base station. This approach will distribute the energy load evenly among the sensor nodes in the network. We initially place the nodes randomly in the play field, and therefore, the $i$-th node is at a random location. The node will be organized to form a chain, which can either be accomplished by the sensor nodes themselves using a greedy algorithm starting from some node. Alternatively, the BS can compute this. We begin with this node in order to make sure that nodes farther from the BS have close neighbours, as in the greedy algorithm the neighbour distances will increase gradually since nodes already on the chain cannot be revisited. A figure 3 shows node 0 connecting to node 3 , node 3 connecting to node 1 , and node 1 connecting to node 2 in that order. when a node dies, the chain is reconstructed in the same manner to bypass the dead node.

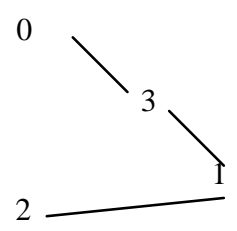

\section{Fig 3: Chain construction using greedy algorithm}

For gathering data in each round, each node receives data from one neighbour, fuses with its own data, and transmits to the other neighbour on the chain. Node co will pass its data towards node $\mathrm{c} 2$. After node $\mathrm{c} 2$ receives data from node $\mathrm{cl}$, it will pass the token to node $\mathrm{c} 4$, and node $\mathrm{c} 4$ will pass its data towards node $\mathrm{c} 2$.

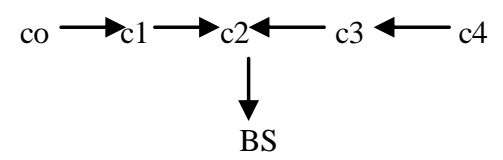

Fig 4. Token passing approach

\subsection{Self Organizing Protocol (SOP):}

Subramanian et al. [21] describes a self-organizing protocol and an application taxonomy that was used to build architecture used to support heterogeneous sensors.

Furthermore, these sensors can be mobile or stationary. Some sensors probe the environment and forward the data to a designated set of nodes that act as routers. Router nodes are stationary and form the backbone for communication. Collected data are forwarded through the routers to the more powerful BS nodes. Each sensing node should be able to reach a router in order to be part of the network. The routing architecture is hierarchical where groups of nodes are formed and merge when needed. Local Markov Loops (LML) algorithm, which performs a random walk on spanning trees of a graph, [20] was used to support fault tolerance and as a means of broadcasting.In this approach, sensor nodes can be addressed individually in the routing architecture, and hence it is suitable for applications where communication to a particular node is required. Furthermore, this algorithm incurs a small cost for maintaining routing tables and keeping a balanced routing hierarchy. It was also found that the energy consumed for broadcasting a message is less than that consumed in the SPIN protocol.

\subsection{Randomized approach}

Energy aware random asynchronous wakeup (RAW-E) protocol [22], a novel cross layer power management and routing protocol for heterogeneous wireless sensor and actor networks, RAW-E is a distributed, randomized algorithm where nodes make local decision on whether to sleep or to be active based on the energy level of its neighbors. The 
primarily result of of RAW-E is the reduction of energy disparity among nodes. Therefore, while the energy reduction is spread uniformly among nodes, the life of network connectivity can be increased. RAW is scalable to change in network size, node type, node density and topology. RAW take advantage of actor nodes, and uses their resources when possible, thus reducing energy consumption of sensor nodes. The performance of this protocol is even good in large network and even scalable with density.

\section{PROPOSED MODEL}

An overview of protocols proposed for heterogeneous networks is given in the table 1 . These protocols need to be improved further or new protocols should be developed to address. We can extend these protocols to deal with more than three types of nodes and to include more than two level of hierarchy.

Important issues/factors that can be explored in these models where the heterogeneity among sensor nodes is not only in their available energy, but also in their processing capabilities and even in energy consumption in their data processing (compression, fusion) etc

Future work could explore similar issues in query driven and event driven types of sensor networks and even multi hop clustering and fault tolerant mechanism could be used in heterogeneous sensor networks.

Future work could explore similar issues in query driven and event driven types of sensor networks and even multi hop clustering and fault tolerant mechanism could be used in heterogeneous sensor networks.

Table 1: Classification of protocols with attributes
On the other hand, cluster-based routing protocols group sensor nodes to efficiently relay the sensed data to the sink. The cluster heads are sometimes chosen as specialized nodes that are less energy-constrained. The most interesting research issue regarding such protocols is how to form the clusters so that the energy consumption and contemporary communication metrics such as latency are optimized. The factors affecting cluster formation and cluster-head communication are open issues for future research. Furthermore, various energy conserving protocols have been highlighted. Although many of these protocols look promising, there are still many challenges and issues to be solved.

Moreover, the process of data aggregation and fusion among clusters is also an interesting problem to explore. Other possible future research for routing protocols includes the integration of sensor networks with wired networks (i.e. Internet).

Although the performance of these protocols is promising in terms of energy efficiency, further research would be needed to address issues such as quality of service posed by video and imaging sensors and real-time applications.

\section{CONCLUSION}

In this article we have given a comprehensive survey of heterogeneous network in wireless sensor models. Throughout the paper efficient use of energy is given top priority. Various techniques under cluster based approach, chain based approach have been discussed to improve network life time, deployment cost, stability and throughput factors.

Comparison analyses of more heterogeneous protocols have been discussed in table 2 .

\begin{tabular}{|l|l|l|l|l|l|l|l|l|l|l|l|}
\hline Protocols & $\begin{array}{l}\text { Classific } \\
\text { ation }\end{array}$ & Mobility & $\begin{array}{l}\text { Position } \\
\text { Awaren } \\
\text { ess }\end{array}$ & $\begin{array}{l}\text { Power } \\
\text { Usage }\end{array}$ & $\begin{array}{l}\text { Data } \\
\text { Aggrega } \\
\text { tion }\end{array}$ & $\begin{array}{l}\text { Localiza } \\
\text { tion }\end{array}$ & QoS & $\begin{array}{l}\text { State } \\
\text { Comple } \\
\text { xity }\end{array}$ & $\begin{array}{l}\text { Scalabili } \\
\text { ty }\end{array}$ & $\begin{array}{l}\text { Multipat } \\
\text { h }\end{array}$ & $\begin{array}{l}\text { Query } \\
\text { based }\end{array}$ \\
\hline PEGASIS & Hierarchal & $\begin{array}{l}\text { Fixed } \\
\text { Base } \\
\text { Station }\end{array}$ & No & Max & No & Yes & No & Low & Good & No & No \\
\hline LEACH & Hierarchal & $\begin{array}{l}\text { Fixed } \\
\text { Base } \\
\text { Station }\end{array}$ & No & Max & Yes & Yes & No & CH & Good & No & No \\
\hline SOP & Hierarchal & $\begin{array}{l}\text { Fixed } \\
\text { Base } \\
\text { Station }\end{array}$ & No & Max & Yes & Yes & No & CH & Good & No & No \\
\hline RAW-E & Flat & $\begin{array}{l}\text { Yo } \\
\text { Yes }\end{array}$ & No & Nes & Yes & Yes & Yes & Yes & Low & Good & Yes \\
\hline
\end{tabular}




\section{REFERENCES}

[1] W.R Heinzelman, Application -Specific Protocol Architectures for Wireless networks, $\mathrm{PhD}$ thesis, Massachusetts institute of Technology,2000

[2] Dilip Kumar and R.B Patel "Prolonging network lifetime and data accumulation in heterogeneous sensor network" International Arab Journal of Information Technology Vol.7,No.3,July 2010.

[3] M.Yarnis, N.Kushalnagar, H.Singh "Exploring Heterogeneity in Sensor networks", IEEE infocomm, 2005

[4] Jun Zheng, Abbas Jamalipour "Wireless Sensor Network: A Network Perspective", IEEE 2009 A JOHN WILEY \& SONS, INC., PUBLICATION

[5] W. Heinzelman, A. Chandrakasan and H.Balakrishnan , "An Application-Specific Protocol Architecture for Wireless Micro sensor Networks," IEEE Transactions on Wireless Communications, Vol. 1, No. 4, 660-670, October 2002.

[6] SH. Lee, JJ. Yoo and TC. Chuan, "Distance-based Energy Efficient Clustering for Wireless Sensor Networks", Proc. of the $29^{\text {th }}$ IEEE Int'l Conf. on Local Computer Networks (LCN'04).

[7] O.Younis and S. Fahmy, "Distributed Clustering in Adhoc Sensor Networks: A Hybrid, Energy-Efficient Approach", In Proc. of IEEE INFOCOM, March 2004.

[8] J. M. Kim, S. H. Park, Y. J. Han, and T. M. Chung, "CHEF: Cluster Head Election mechansim using Fuzzy logic in Wireless Sensor Networks", Proc. of ICACT, 654-659, Feb. 2008.

[9] Yingchi Mao, Zhen Liu, Lili Zhang, Xiaofang Li, "An Effective Data Gathering Scheme in Heterogeneous Energy Wireless Sensor Networks," cse, vol. 1, pp.338343, 2009 International Conference on Computational science and Engineering, 2009.

[10] Dilip Kumar, T. S. Aseri, R. B. Patel "EEHC: Energy efficient heterogeneous clustered scheme for wireless sensor networks", International Journal of Computer Communications, Elsevier, 2008, 32(4): 662-667, March 2009.

11] Dilip Kumar, T. S. Aseri, R.B Patel "EECHE: Energy Efficient Cluster head election protocol for heterogeneous WirelessSensor Networks," in Proceedings of ACM International Conference on
Computing, Communication and Control-09 (ICAC3'09), Bandra, Mumbai, India, 23-24 January 2009, pp. 75-80.

[12] M. Golam Rashed, M.Hasnat Kabir, Shaikh Enayet Ullah "Cluster Based Hierarchal Routing Protocol For WSN" IJCNS, Vol.2, No.5, May2010.

[13] Boseung Kim, Joohyun Lee, Youngtae Shin "RCFT: ReClustering Formation Technique in Hierarchal Sensor Network", IJCSIS, Vol.6, No. 1, 2009.

[14] S.Lindsey and C.Raghavendra "PEGASIS: Power efficient gathering in sensor info system", conf.Proc.2002, vol 3, 9-16 pp. 1125-30.

[15] G.Samargdakis, I.Matta, A.Bestavros 2004, "SEP: A stable Election Protocol for clustered Heterogeneous wireless sensor network", In second international workshop on sensor and actor network protocols and application (SANPA).

[16] I.Akyilidiz et al 2002 "A survey on sensor networks" ,IEEE communication Magazine ,vol 40,no. 8,pp. 10214.

[17] Samayveer Singh, Ajay K Sharma "Energy-Efficient Data Gathering Algorithms for Improving Lifetime of WSNs with Heterogeneity and Adjustable Sensing Range",International Journal of Computer Applications (0975 - 8887) Volume 4 - No.2, July 2010

[18] Ming Liu, Jiannong Cao, Guihai Chen 3 and Xiaomin Wang "An Energy-Aware Routing Protocol in Wireless Sensor”, ISSN 1424-8220, Networks, SENSORS 2009

[19] S. Dulman, T. Nieberg, J. Wu, P. Havinga, ITrade-Off between Trafic Overhead and reliability in Multipath Routing for Wireless Sensor etworks", WCNC Workshop, New Orleans, Louisiana, USA, March 2003

[20] ZhouHaibo, WuYuanming ,XieGuangzong, "EDFM:Stable election protocol based on energy dissipation forecast method for clustering heterogeneous wsn", Proceeding in IEEE 2009.

[21] L. Subramanian and R. H. Katz, "An architecture for Building Self Confgurable Systems", in the Proceedings of IEEE/ACM Workshop on Mobile Ad Hoc Networking and Computing, Boston, MA, August 2000.

[22]Vamsi Paruchuri,Arjan Durresi ,Leonard Baroli , "Energy aware Routing protocol for heterogeneous wireless Sensor networks", Proceeding of $16^{\text {th }}$ international workshop on database and expert system, IEEE 2005. 
Table 2: Heterogeneous protocols comparison based on parameters

\begin{tabular}{|c|c|c|c|c|c|c|c|}
\hline Approach & $\begin{array}{l}\text { Node } \\
\text { Mobility }\end{array}$ & $\begin{array}{l}\text { Cluster } \\
\text { Overlapping }\end{array}$ & $\begin{array}{l}\text { Location } \\
\text { Awareness }\end{array}$ & Energy efficient & $\begin{array}{l}\text { Failure } \\
\text { Recovery }\end{array}$ & $\begin{array}{l}\text { Balanced } \\
\text { Clustering }\end{array}$ & $\begin{array}{l}\text { Cluster } \\
\text { stability }\end{array}$ \\
\hline LEACH & Fixed BS & No & Not Required & & Yes & Ok & Moderate \\
\hline HEED & Stationary & No & Not Required & Yes & N/A & Good & High \\
\hline $\begin{array}{l}\text { Hierarchal } \\
\text { clustering }\end{array}$ & Possible & Low & Not Required & N/A & Yes & Good & Moderate \\
\hline EEHC & No & No & Required & Yes & N/A & Ok & N/A \\
\hline RCFT & Possible & No & Required & Yes & Yes & Good & High \\
\hline CODA & Possible & No & Required & $\begin{array}{l}\text { Improvement on } \\
\text { LEACH }\end{array}$ & Yes & Ok & N/A \\
\hline EDGA & Fixed BS & No & Not Required & Yes & Yes & Ok & N/A \\
\hline CBRP & Fixed BS & Yes & Required & Yes & yes & Ok & Moderate \\
\hline CHEF & Fixed BS & No & yes & Yes & Yes & Ok & Moderate \\
\hline $\mathrm{RCC}$ & Yes & No & Required & N/A & Yes` & Good & Moderate \\
\hline EDFM & Fixed BS & yes & required & Yes & Yes & Good & High \\
\hline
\end{tabular}

